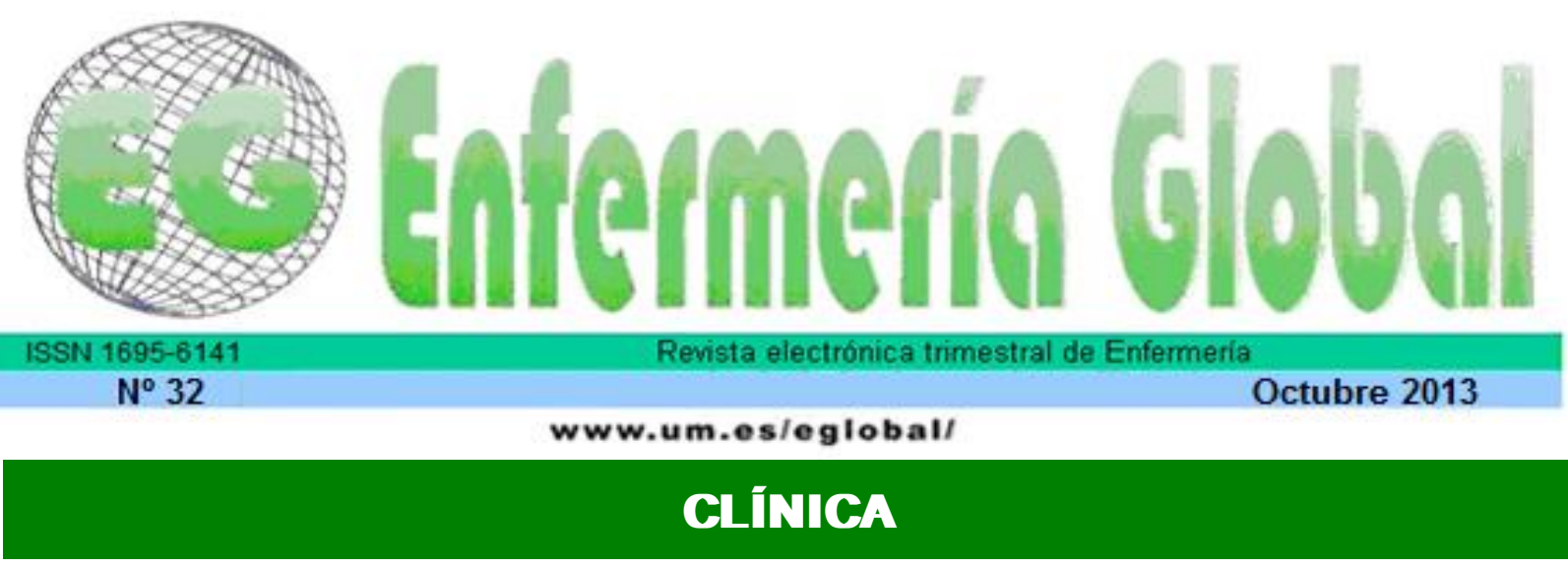

\title{
Conocimiento del equipo de enfermería sobre la inserción y mantenimiento del catéter central de inserción periférica en recién nacidos
}

Conhecimento da equipe de enfermagem sobre inserção e manutenção do cateter central de inserção periférica em recém nascidos

Knowledge of the team of nursing on insertion and maintenance of the central catheter of peripheral insertion in just born

\section{*Silva Bretas, Tereza Cristina **Silva Fagundes, Magna Ferreira *** Versiani, Clara de Cássia ****Marques Andrade, Frederico.}

\begin{abstract}
*Enfermera, Especialista, Profesora del Curso de Graduación en Enfermería. E-mail: cabela@ig.com.br $\quad{ }^{* *}$ Enfermera. ***Máster en Enfermería. Profesora del Curso de Graduación en Enfermería. ****Enfermero, Especialista, Profesor del Curso de Graduación en Enfermería. Universidad Estadual de Montes Claros (UNIMONTES). Brasil.
\end{abstract}

\begin{abstract}
Palabras clave: Enfermería; Unidad de terapia intensiva neonatal; educación en salud.
Palavras chave: Enfermagem; Unidade de Terapia Intensiva Neonatal; educação em saúde Keywords: Nursing; Intensive Care Units Newborn; education in health.
\end{abstract}

\section{RESUMEN}

Objetivo: Evaluar el conocimiento del equipo de enfermería sobre la inserción y manipulación del PICC.

Método: Estudio descriptivo, documental y de campo. El escenario fue la Unidad de Cuidados Intensivos Neonatal (UTIN) del Hospital Universitario Clemente de Faria, de Montes Claros, Minas Gerais, Brasil entre julio y diciembre de 2011. Participaron 48 profesionales de enfermería.

Resultados: Se comprobó que una parte de los profesionales desconocen la definición correcta del PICC, tienen dudas sobre la vena de primera elección para la punción, período de permanencia, tipo de jeringa a ser utilizada y permeabilización del catéter.

Conclusión: La formación contínua es capaz de llenar las lagunas, formar parte del proceso de trabajo, proporcionar reflexión y actualización de las prácticas en el cotidiano del profesional. 


\section{RESUMO}

Objetivo: Avaliar o conhecimento da equipe de enfermagem sobre a inserção e o manuseio do PICC.

Método: Estudo descritivo, documental e de campo. O cenário foi a UTI Neonatal do HUCF, de julho a dezembro de 2011. Fizeram parte do estudo 48 profissionais da enfermagem.

Resultado: Observa-se que parte dos profissionais desconhece a definição correta de PICC, têm dúvidas sobre a veia de primeira escolha para punção, período de permanência, seringa a ser utilizada e permeabilização do cateter.

Conclusão: A educação permanente é capaz de suprir lacunas, fazer parte do processo de trabalho, proporcionar reflexão e atualização das práticas no cotidiano do trabalhador.

\section{ABSTRACT}

Objective: To evaluate the knowledge of the nursing team on the insertion and the handling of the PICC.

Method: Descriptive, documentary and field study. The locale was the ICU Newborn of the HUCF, July to December of 2011. 48 professionals of the nursing staff were part of the study.

Results: It was observed that part of the professionals are unaware of the correct definition of PICC, have doubts on the vein of first choice for punction, period of permanence, syringe to be used and permeabilization of the catheter.

Conclusion: The permanent education is able to fill the gaps, to be part of the work process, to provide reflection and updating of the daily practices of the worker.

\section{INTRODUCCIÓN}

En la actualidad, con el creciente desarrollo científico-técnico y los avances tecnológicos alcanzados el perfil de los niños ingresados en las Unidades de Terapia Intensiva Neonatal (UTIN) ha experimentado modificaciones considerables, demandando así cuidados más complejos tales como la utilización de procedimientos invasivos para garantizar su supervivencia ${ }^{(1,2)}$. Tales avances tecnológicos en las UTIN han proporcionado muchos beneficios para los recién nacidos logrando un aumento significativo en la supervivencia de los mismos ${ }^{(4)}$. Se verifica así el surgimiento de nuevas técnicas, y con ello el perfeccionamiento de los cuidados de alto riesgo ofrecidos a los mismos. Entre ellos, la terapia intravenosa resulta de extraordinaria importancia para la asistencia a dichos neonatos.

Entre las nuevas técnicas y dispositivos ha surgido el Catéter Céntral de Inserción Periférica (PICC). Se trata de un dispositivo intravenoso que es insertado a través de una vena superficial de la extremidad y que progresa, por medio de una aguja y del flujo sanguíneo, hasta el tercio distal de la vena cava superior o inferior, adquiriendo características de un catéter central ${ }^{(5,6)}$.

La obtención de un acceso venoso seguro representa uno de los mayores desafíos para que el equipo pueda implementar la terapia medicamentosa, asegurar el tratamiento y la calidad de la asistencia ${ }^{(1)}$

El PICC es considerado por muchos autores un dispositivo de acceso vascular seguro. Está indicado para las terapias de duración prolongada (por más de una semana), la administración parenteral con concentración de dextrosa superior al 10\%, 
para disminuir el número de punciones, lograr el acceso venoso central para la administración de drogas irritantes o vesicantes ${ }^{(5)}$, la prevención de flebitis 0 quemaduras debido a extravasaciones, disminuir el dolor causado por las consecutivas punciones, entre otros ${ }^{(7)}$; de soluciones hiperosmolares o con el pH no fisiológico, y cuando sea necesario monitorear la presión venosa central ${ }^{(5)}$.

Su uso está contraindicado en recién nacidos (RN) con lesiones cutáneas en el lugar de la inserción, en la administración de grandes volúmenes en bolus o bajo presión, cuando el retorno venoso esté perjudicado, en caso de emergencias o cuando los familiares rechacen aceptar tal procedimiento ${ }^{(5)}$.

Con la utilización del PICC los recién nacidos tienen la oportunidad de realizar la terapéutica intravenosa, disminuyendo el estrés causado por las sucesivas punciones, favoreciendo la infusión de soluciones de diferentes concentraciones con un riesgo menor de iatrogenias ${ }^{(3)}$, sustituyendo las flebotomías con gran éxito, y por tener la disponibilidad de diámetros muy reducidos, es posible utilizar esa técnica en lactantes y recién nacidos de bajo peso que necesitan de un acceso venoso prolongado o con fragilidad venosa durante el período de ingreso hospitalario ${ }^{(8)}$.

En Brasil, la atribución de la competencia técnica y legal para la inserción y manipulación del PICC por el Enfermero se encuentra amparada por la Resolución $n^{\circ}$ 258 de 2011 del Consejo Federal de Enfermería (COFEN) ${ }^{(3)}$.

La inserción del PICC es un procedimiento de gran complejidad técnica y exige conocimientos específicos ${ }^{(5)}$. Es importante que los enfermeros que realizan dicho procedimiento sean capacitados y entrenados, para adquirir los conocimientos necesarios con relación a las indicaciones del uso de los catéteres, a los procedimientos adecuados para la inserción y el mantenimiento de dichos dispositivos, así como de las medidas correctas del control de las complicaciones mecánicas e infecciosas relacionadas con los mismos ${ }^{(1,6)}$.

Al considerar que los cuidados del paciente con PICC presupone aspectos peculiares para el mantenimiento de tal dispositivo, a fin de asegurar su permanencia y la disminución de las complicaciones con el mismo, se percibió la necesidad de desarrollar este estudio de carácter educativo, que tuvo como objetivos reflexionar sobre el papel del profesional de enfermería en los cuidados del paciente con PICC desde una visión tecnológica ${ }^{(9)}$.

\section{MATERIALES Y MÉTODOS}

Este es un estudio exploratorio, descriptivo, documental y de campo, con abordaje cuantitativo. El escenario de esta investigación fue la UTIN del HUCF. Para la realización de la misma se optó por un abordaje censitario, o sea, se trabajó con el universo de los sujetos. Por eso fueron incluídos los miembros del equipo de enfermería de la UTIN, con experiencia de actuación superior a un año en dicho sector y firmantes del Término de Libre Consentimiento y Consciencia. Formaron parte del presente estudio 48 profesionales de enfermería (entre enfermeros y técnicos de enfermería). Para el levantamiento de los datos primarios se aplicó a los participantes una encuesta adaptada del cuestionario validado ${ }^{(8)}$, por medio de entrevistas. Las informaciones recolectadas fueron lanzadas en una base de datos del Software SPSS (Satistical Package sea the Social Sciences) versión 16. 0 para Windows, viabilizando la construcción de las tablas. Las descripciones realizadas por 
medidas de frecuencia, promedios y desvíos normalizados para las variables numéricas y proporción en tantos por cientos (\%) para las variables categóricas. Los aspectos éticos de la investigación están en consonancia con la Resolución n 196 de 10/10/96 de la Comisión Nacional de Ética en Investigación del Ministerio de la Salud. La investigación fue aprobada mediante el parecer número 3113 por el Comité de Ética en Investigación (CEP) de la Universidad Estadual de Montes Claros (UNIMONTES).

\section{RESULTADOS Y DISCUSIÓN}

Formaron parte del presente estudio 48 profesionales de enfermería, 6 enfermeros, un $12,5 \%$ y 42 técnicos de enfermería, un $87,5 \%$. Destacar la importancia del equipo de enfermería y, sobre todo, del profesional enfermero, pues este es en la realidad brasileña uno de los principales responsables de la indicación del uso de ese catéter, además de estar directamente involucrado en la inserción y en las soluciones a las complicaciones relacionadas al PICC ${ }^{(10)}$.

El promedio de tiempo de servicio de los sujetos fue de 5,7 años (con un mínimo de 1 año y un máximo de 17 años). Con relación al tiempo de servicio en el sector resulta de gran importancia pues con el paso del tiempo el profesional adquiere un mayor conocimiento teórico y práctico sobre los cuidados a ser realizados, ampliando sus conocimientos con su vivencia profesional y experiencia adquirida ${ }^{(9)}$.

Es muy importante que exista un incentivo constante a estos profesionales para mantener el entusiasmo dentro de un sector que es de alta complejidad, para que lo técnico no se sobreponga a lo humano ${ }^{(11)}$.

Debido a que la inserción del PICC es un procedimiento realizado sólo por los enfermeros, algunas preguntas del cuestionario fueron dirigidas exclusivamente a ellos. Tales cuestiones tenían como próposito evaluar el conocimiento de los enfermeros sobre la técnica de inserción del PICC. Así, fueron encuestados sobre cuáles serían los vasos de primera elección para la inserción del PICC (fig. 1), de ellos un 66,7\% dijo ser la vena basílica, cefálica y cubital mientras que un 33,3\% respondió que era la vena basílica, grande safena y cubital.

La utilización del PICC posee algunas peculiaridades que van desde la elección del vaso hasta el mantenimiento del mismo. La vena basílica resulta ser la más utilizada, seguida de la vena cefálica. Por lo tanto, se nota que hay algún desconocimiento sobre las venas de elección ${ }^{(11)}$. 
Figura 1. Gráfico que representa la primera elección de las venas para la inserción del PICC de acuerdo con la visión de los enfermeros de la UTIN.

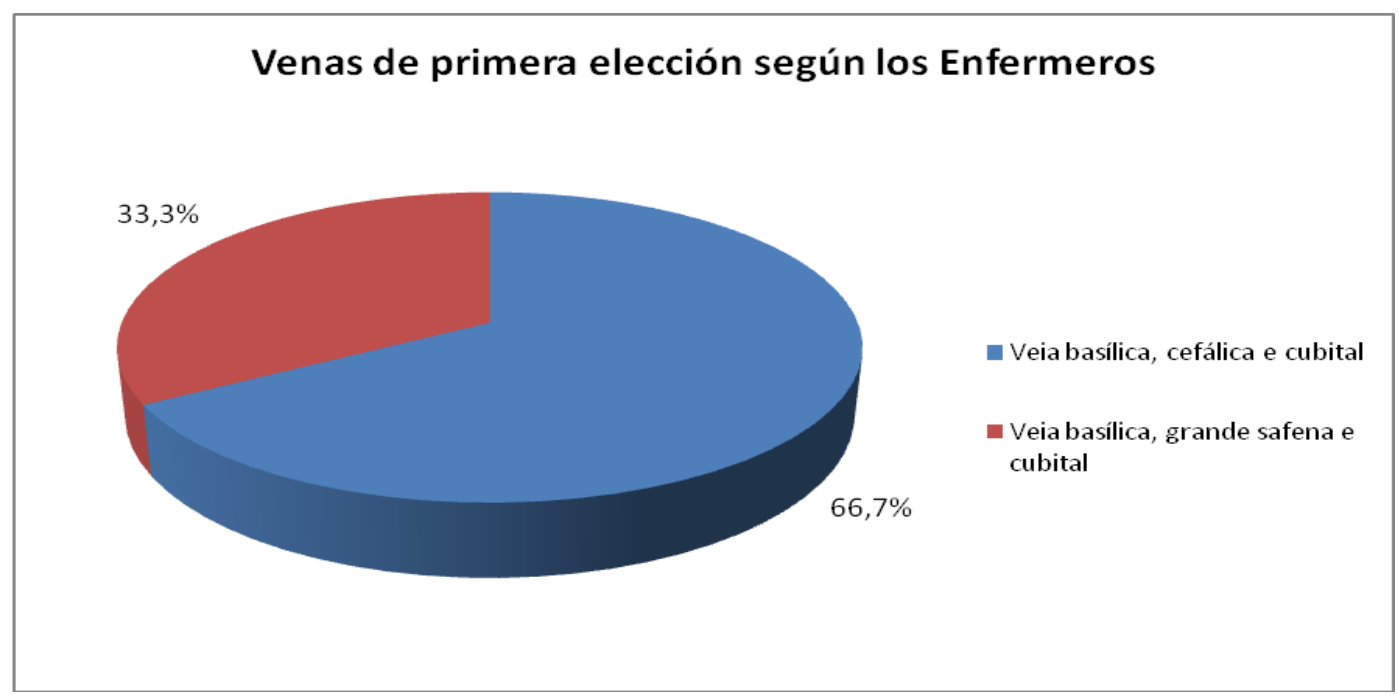

Fuente: UTIN HUCF, Montes Claros, MG, Brasil, 2011

También fueron interrogados si para la utilización del PICC era necesario orientar a la familia sobre el mismo y si para la inserción del PICC y ejecución de la primera cura era necesario utilizar una "barrera estéril" (usar campos quirúrgicos, delantales de manga larga, guantes estérilizados, máscara, cofia), todos los enfermeros respondieron afirmativamente a las dos preguntas.

Al analizar la Tabla I, se puede observar que parte de los profesionales desconocen la definición correcta de PICC, pues el 35,4\% respondió que PICC es el Catéter Periférico de Inserción Central y un profesional no sabía el significado de la sigla. El término PICC hace alusión al término en lengua inglesa" Peripherally Inserted Céntrico Catheters" (Catéter Central de Inserción Períferica) ${ }^{(3,12)}$. En cuanto a su importancia, la mayoría - un 97,9\%- respondió que es evitar que el recién nacido sufra con reiteradas punciones periféricas. También se les preguntó sobre las ventajas e indicaciones del PICC. Referente a las ventajas, el 87,5\% respondió que era "evitar las múltiples punciones venosas y preservar el sistema venoso periférico". $\mathrm{Ya}$, al tratarse de las indicaciones - la mayor parte,el 95,8\%- dijo que era suministrar las soluciones hiperosmolares.

Algunas ventajas en la utilización del PICC: la inserción del catéter bajo anestesia local, la reducción de la incomodidad del paciente, toda vez que este no será sometido a incontables punciones, el hecho de poder ser insertado por un profesional enfermero a pie de cama, por ser una vía confiable para la administración de quimioterápicos y la nutrición parenteral ${ }^{(5)}$.

EI PICC permite la administración de fluídos y medicamentos que no pueden ser infundidos en las venas periféricas. Tiene como indicaciones el uso de terapias prolongadas (superiores a los 7 días), la administración de la nutrición parenteral con concentración de dextrosa mayor del 10\%, la infusión de medicamentos vesicantes, irritantes, vasoactivos e hiperosmolares.

Como contraindicación tiene: la administración de grandes volúmenes en bolus, la infección en el local de la inserción, el retorno venoso perjudicado, la trombosis venosa $^{(5)}$. 
Se resalta que los vasos delgados, de difícil identificación, comprometidos por edema, eritema y hematomas (causados por repetidas punciones venosas o cirugías traumáticas), constituyen los mayores problemas para la inserción y el mantenimiento del catéter. La indicación debe ser precoz, siendo la primera elección. Por eso, el dispositivo no es recomendado para todos los pacientes. Cabe al enfermero, conjuntamente con el equipo médico, evaluar e indicar o no la inserción del PICC ${ }^{(12)}$.

Sobre el período de permanencia del mismo, el 87,0\% respondió que es un catéter de larga permanencia mientras el 10,9\% que su utilización se debe dar hasta el término del tratamiento. Referente al tiempo de permanencia, el PICC es indicado para el RN que necesite la terapia intravenosa por un período superior a los seis días, siendo que el tiempo de permanencia es de ocho semanas como promedio ${ }^{(12)}$.

En cuanto a la jeringa que no se debe utilizar para manipular el PICC, el $87,5 \%$ dijo que era la jeringa de $1 \mathrm{ml}$ e igual número el 6,3\% dijo ser las jeringas de 10 y $20 \mathrm{ml}$. La salinización del catéter debe ser hecha con una jeringa de $10 \mathrm{ml}$, que tiene una presión menor, reduciendo el riesgo de rotura accidental del mismo ${ }^{(13)}$.

Se deben usar solamente las superiores a $5 \mathrm{ml}$, pues, cuanto menor sea la jeringa, mayor será la presión, lo que puede causar la ruptura del catéter ${ }^{(7,10)}$. Se preguntó también a los profesionales si el PICC puede ser permeabilizado, y el $87,5 \%$ dijo que tal práctica es adecuada mientras el $12,5 \%$ respondió que no.

Tabla I: Conocimiento de los profesionales de enfermería en cuanto a la definición, la importancia, las ventajas, las indicaciones, el período de permanencia, el uso de la jeringa para manipular el catéter y la permeabilización del mismo.

\begin{tabular}{lcr} 
Variable & $\mathrm{n}$ & \\
\hline Qué es el PICC & & \\
\hline Catéter Central de Inserción Periférica & 30 & $62,5 \%$ \\
Catéter Periférico de Inserción Central & 17 & $35,4 \%$ \\
No sabe & 01 & $2,1 \%$ \\
Importancia del PICC en la UTIN & 47 & $97,9 \%$ \\
Evita que el RN sufra con punciones periféricas & 01 & $2,1 \%$ \\
Mantener el RN con un acceso venoso que facilita la hemotransfusión & & \\
Ventajas del PICC & 42 & $87,5 \%$ \\
Evita las múltiples punciones venosas y preserva el sistema venoso periférico & 06 & $12,5 \%$ \\
Es más rápido e insertado por enfermeros & & \\
Indicaciones del PICC & 46 & $87,5 \%$ \\
Administrar las soluciones hiperosmolares & 01 & $2,1 \%$ \\
La terapia venosa por menos de 7 días & 01 & $2,1 \%$ \\
Previene la trombosis venosa & & \\
Cómo es el período de permanencia del PICC & 42 & $87,5 \%$ \\
De larga permanencia & 01 & $6,3 \%$ \\
De corta permanencia & 01 & $6,3 \%$ \\
Hasta el fin del tratamiento & & \\
Jeringa que no puede ser usada para manosear el PICC & 42 & $87,5 \%$ \\
La jeringa de 1 ml & $6,3 \%$ \\
La jeringa de 10ml & 03 & $6,3 \%$ \\
La jeringa de 20ml & 03 & \\
Necesidad de permeabilización del catéter & & $87,5 \%$ \\
Es necesario & 42 & $12,5 \%$ \\
No es necesario & 06 & $100 \%$ \\
Número total & 48 &
\end{tabular}

Fuente: UTIN HUCF, Montes Claros, MG, Brasil, 2011 
Otra cuestión discutida fue sobre la utilización de los hemoderivados en el PICC (fig.2). Al analizar los datos se observa que 42 profesionales o sea el $87,2 \%$ respondió no ser correcta la utilización del PICC para la administración de los hemoderivados, pero seis, el 12,8\%, dijo ser adecuada la utilización de los mismos para tal procedimiento.

Las referencias bibliográficas sobre el tema afirman que la infusión de los hemoderivados, los hemocomponentes y la recolección de sangre deben hacerse con catéteres de diámetro superior a los 3,8 French, para que no ocurra una obstrucción $(5,10,13,14)$.

Figura 2. Conocimiento de los profesionales de Enfermería sobre la utilización de los hemoderivados en el PICC.

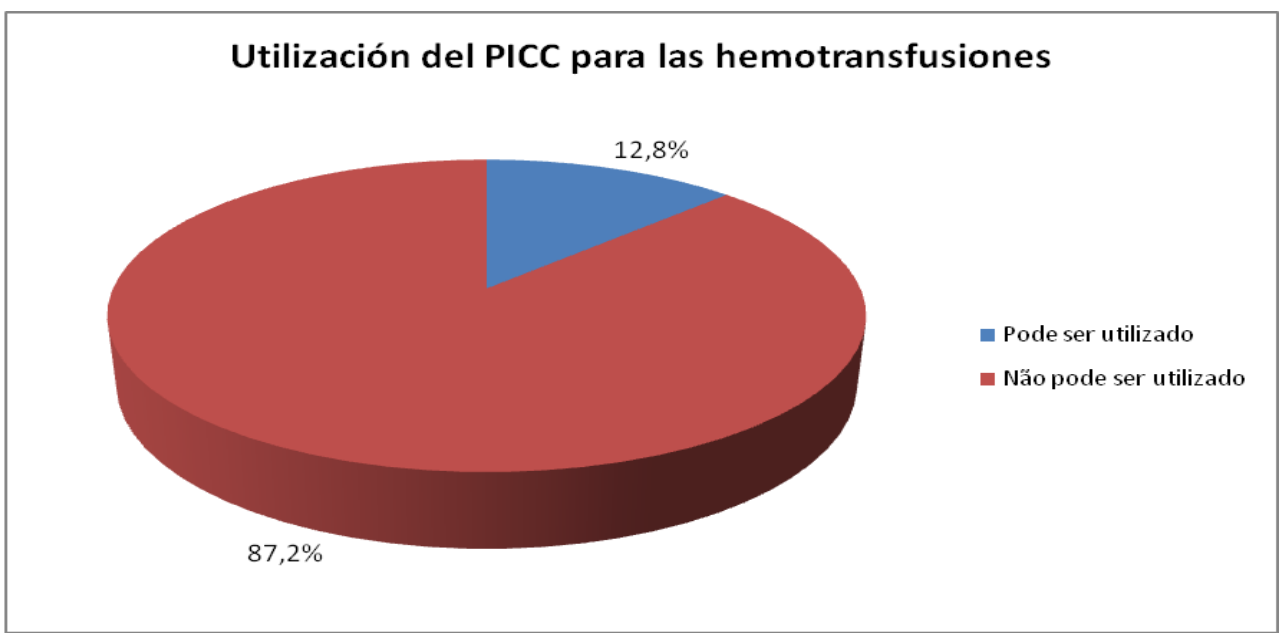

Fuente: UTIN HUCF, Montes Claros, MG, Brasil, 2011

Durante la práctica, a veces, el PICC suele obstruirse. Frente a esto, fueron preguntados los profesionales con relación al procedimiento correcto en tal situación, siendo que el 54,3\% respondió que no se deben hacer maniobras de desobstrución, el 32,6\% que la desobstrucción debe ser realizada con una solución salina al 0,9\% mientras que el 13,0\% afirmó que se debe usar el ABD en esa situación (fig. 3).

Debe destacarse que el método de salinización administración del medicamento salinización y heparinización (SASH) es aconsejado y previene la ocurrencia de la incompatibilidad entre las soluciones transfundidas, además de mantener el buen funcionamiento del catéter. El volumen de solución salina varía entre 10 y $20 \mathrm{ml}$ después de la transfusión de soluciones hiperosmolares, medicamentos vesicantes e irritantes, hemoderivados o recolección de muestra sanguínea, tamaño del catéter y edad del niño ${ }^{(10)}$.

Los PICC no son artefactos exentos de complicaciones y entre ellas - a veces- suele ocurrir la obstrucción, siendo la más común la causada por depósitos de fibrina y componentes de la sangre.

La prevención de las oclusiones trombóticas incluye desde el lavado frecuente del catéter, hasta el cuidado para evitar el reflujo de sangre para la penetración durante la manipulación. Los intentos para desobstruir los catéteres siempre llevan aparejado el riesgo de ruptura, aún cuando son utilizados trombolíticos, pues existe el problema de transfundirse una solución en un catéter que esté totalmente obstruído ${ }^{(15)}$. 
Se observa que los participantes reconocen la necesidad de cuidados específicos con el PICC, y que la permanencia del mismo y las posibles complicaciones están relacionadas al mantenimiento adecuado del dispositivo ${ }^{(10)}$.

Por último, los profesionales fueron preguntados sobre la realización de entrenamientos con ellos y sobre la existencia del protocolo en la institución que trata del uso del PICC. Más de la mitad de los entrevistados - el 62,5\%- dijo no haber recibido entrenamiento sobre la inserción y/o mantenimiento del PICC. Es muy importante que los profesionales de enfermería que trabajan en las unidades de terapia intensiva neonatal sean capacitados para el paso del PICC, su mantenimiento y la prevención de sus complicaciones ${ }^{(1,3,17,18)}$.

Se considera que la sensibilización, el compromiso y la valorización del equipo de enfermería en lo concerniente al cuidado del paciente con PICC, desde la visión tecnológica, contribuyen a la reflexión sobre la práctica y la disminución de las pérdidas del dispositivo.

En cuanto a la existencia de protocolo el $66,0 \%$ respondió que la institución lo posee mientras el $34,0 \%$ que no. Se comprobó cierto desconocimiento por parte de los profesionales con relación a la existencia de un protocolo institucional.

En estudio realizado fue observado que después de la elaboración y la operacionalización del protocolo del catéter hubo una mejoría de la calidad del cuidado prestado en la asistencia ${ }^{(10)}$.

\section{CONCLUSIÓN}

El presente trabajo permitió verificar el conocimiento teórico y práctico de los profesionales de Enfermería. Se observa que los enfermeros de la institución tienen un buen conocimiento referente al proceso de inserción del PICC, sin embargo, al considerar la todalidad de los profesionales de enfermería, se verifica la existencia de cierto desconocimiento por parte del equipo en algunas cuestiones.

Los participantes reconocieron la necesidad de una formación permanente, lo que propicia la discusión sobre el cuidado de enfermería al paciente con catéter PICC. Debido a la especificidad del catéter, este tipo de educación en los servicios de salud es capaz de suplir lagunas, así como de formar parte del proceso de trabajo y proporcionar la reflexión y la actualización de las prácticas en la cotidianeidad del trabajador ${ }^{(10,18)}$.

Se destaca que la Ley GM/MS no 198/2004 instituye la Política Nacional de Educación Permanente en Salud, como estrategia del Sistema Único de Salud (SUS), para la formación y el desarrollo de los profesionales, y busca incrementar el perfeccionamiento individual e institucional ${ }^{(10,16)}$.

Resulta de suma importancia que los profesionales de enfermería tengan conocimientos sobre todos los aspectos relativos al uso del PICC, así como también de los riesgos a los cuales están expuestos los recién nacidos sometidos a tal procedimiento, a fin de utilizar las medidas de prevención, control y detección de las posibles complicaciones consecuentes. 
Aspiramos a que el presente estudio pueda contribuir a la práctica de la Enfermería y fundamentar el desarrollo de actividades que busquen mejorar la formación de los profesionales actuantes en las unidades de terapia intensiva.

\section{BIBLIOGRAFÍA}

1.Gomes, Avo et al. O cateter epucutâneo na unidade de terapia intensiva neonatal: uma tecnologia do cuidado de enfermagem. Enfermería Global, n. 19, 2010.

2.Tamez, RN; Silva, MJP. Enfermagem na UTI neonatal: assistência ao recémnascido de alto risco. $2^{a}$ ed. Rio de Janeiro: Guanabara Koogan; 2002.

3.BRASIL. Ministério da Saúde. Conselho Federal de Enfermagem. Resolução nํ. 258 de 12 de julho de 2001. Inserção de cateter periférico central pelos enfermeiros. Rio de Janeiro; 2001.

4.Montes, SF et al. Ocorrência de complicações relacionadas ao uso de cateter venoso central de inserção periférica (PICC) em recém-nascidos. Enfermería Global, n. 24, 2011.

5.Rodrigues, ZS; Chaves, EMC; Cardoso, MVLML. Atuação do enfermeiro no cuidado com o cateter central de inserção periférica no recém-nascido. Revista Brasileira de Enfermagem, Brasília, v. 59, n. 5, set./out. 2006. Disponível em: http://www.scielo.br/pdf/reben/v59n5/v59n5a06.pdf Acesso: 21 de out. 2011.

6.Phillips LD. Manual de terapia intravenosa. $2^{\underline{a}}$ ed. Porto Alegre (RS): Artmed; 2001. 7.Jesus,VC; Secoli, SR. Complicações acerca do Cateter Venoso Central de Inserção Periférica (PICC). Ciência, cuidado e saúde, v. 6, n. 2, 2007.

8.Teixeira, et al. O conhecimento da equipe de enfermagem sobre o manuseio do cateter central de inserção Periférica- PICC em uma UTIN de um hospital do Sul de minas (monografia apresentada à Universidade José do Rosário Vellano), 2009.

9.Miranda, AM. Epidemiologia do Uso do Cateter Venoso Central em Unidade de Terapia Intensiva Neonatal. 2005. Apresentada como Dissertação de Mestrado em Enfermagem. Universidade Federal de Minas Gerais, Belo Horizonte.

10.Stocco, JGD et al. Cateter central de inserção periférica: percepções da equipe de enfermagem. Cogitare de Enfermagem, v. 16, n. 1, 2011.

11.Baggio, MA; Bazzi, FCS; Bilibio, CAC. Cateter central de inserção periférica: descrição da utilização em UTI Neonatal e Pediátrica. Revista Gaúcha de Enfermagem, v. 31, n.1, 2010.

12.BRASIL. Ministério da Saúde. Conselho Federal de Enfermagem. Resolução no 258 de 12 de julho de 2001. Inserção de cateter periférico central pelos enfermeiros. Rio de Janeiro; 2001. Disponível em: http://site.portalcofen.gov.br/node/4296 Acesso em: 10 de Nov. 2011.

13.Vendramin P. Cateter central de inserção periférica (CCIP). In: Harada MJCS, Rego RC, Orgs. Manual de terapia intravenosa em pediatria. São Paulo: ELLU; 2005. cap. 7, p.75-95.

14.Araújo, OR et al. Heparina intermitente não é eficaz em impedir a retirada por obstrução de cateteres centrais inseridos perifericamente em recém-nascidos de termo e prematuros. Revista Brasileira de Terapia Intensiva, v. 23, n. 3, 2011 Disponível em: http://www.scielo.br/pdf/rbti/v23n3/v23n3a12.pdf Acesso: 27 de nov. 2011.

15.Lino MM, Backes VMS, Schmidt SMS, Ferraz F, Prado ML, Martins T. A realidade da educação continuada na enfermagem nos serviços públicos de saúde de Florianópolis. Online Braz J Nurs. [Internet] 2007;6(0) [acesso em 6 nov 2008]. Disponível: http://www.objnursing.uff.br/index.php/nursing/article/view/619/147.

16.Camargo, PP et al.. Localização inicial da ponta de cateter central de inserção periférica (PICC) em recém-nascidos. Rev. esc. enferm. USP, v. 42, n. 4, 2008. 
17.Ministério da Saúde (BR). Portaria GM/MS n. 198/04, de 13 de fevereiro de 2004. Institui a política Nacional de educação permanente em saúde para formação e o desenvolvimento de trabalhadores para o setor dá outras providências. Diário Oficial da República Federativa do Brasil, Brasília, 14 fev. 2004. Seção 1:1.

18.Câmara, SMC; Tavares, TJL; Chaves, EMC. Cateter venoso de inserção periférica: análise do uso em recém-nascidos de uma unidade neonatal pública em Fortaleza. Revista RENE, v. 8, n.1, 2007. 